\title{
Amitriptyline and Lorazepam Improved Catatonia and Occipital Hypoperfusion in a Patient with DLB
}

\author{
Kengo Maeda and Nobuhiro Ogawa
}

\begin{abstract}
A 76-year-old woman presented with catatonia, refusal to eat due to delusion, and visual hallucination. Single photon emission computed tomography showed remarkable occipital hypoperfusion and frontal hyperperfusion. ${ }^{123}$ I metaiodobenzyl guanidine myocardial scintigraphy revealed decreased uptake. She was diagnosed as probable dementia with Lewy bodies (DLB). Intravenous or oral L-dopa had no effect on catatonia. Amitriptyline and lorazepam improved catatonia and visual hallucination. Cerebral blood flow of the frontal and occipital lobes seemed to be normalized. Occipital hypoperfusion is one of the features of DLB. Although the mechanism of perfusion abnormality in DLB remains to be clarified, our case suggested that it might be reversible.
\end{abstract}

Key words: dementia with Lewy bodies, catatonia, occipital hypoperfusion, depression, antidepressant

(Intern Med 50: 363-366, 2011)

(DOI: 10.2169/internalmedicine.50.4512)

\section{Introduction}

Symptoms of dementia with Lewy bodies (DLB) consist of dementia and parkinsonism. Compared with Alzheimer's disease, patients with DLB show severe visual hallucination and delusion. Neuroimaging using single photon emission tomography (SPECT) or positron emission tomography is useful for the differential diagnosis between Alzheimer's disease and DLB. Hypometabolism and hypoperfusion in the occipital lobe is a characteristic feature of DLB (1). Neurolepic supersensitivity sometimes makes it difficult to treat these symptoms (2). Some studies have shown the usefulness of the acetylcholinesterase inhibitor donepezil on psychiatric symptoms in DLB (3-5). We herein present a woman with probable DLB who showed catatonia and refusal to eat. We treated her with amitriptyline and lorazepam, which dramatically improved her symptoms and normalized occipital cerebral blood flow.

\section{Case Report}

A right-handed 76-year-old woman was admitted to our hospital from another hospital to treat motor and psychiatry symptoms. She had experienced resting tremor at the age of 72. She had been treated with anti-parkinsonian drugs. Within three years, she had complained that some insects developed in her stomach and that she was chased by a large snake. Since she had attempted to remove the insects from her stomach with a scissors and a knife, she was admitted to hospital. She had been treated with strong tranquilizers including chlorpromazine/CPZ or quetiapine. In addition, Yi-Gan San and donepezil had been tried but there had been no effect on psychosis. Since she refused to eat and showed severe akinesia, she was transferred to our hospital. She had been treated with donepezil $(5 \mathrm{mg})$, quetiapine $(150 \mathrm{mg})$, 1-dopa/benserazide $(100 \mathrm{mg})$, amlodipine $(2.5 \mathrm{mg})$, candesartan cilexetil $(4 \mathrm{mg})$, and trichlormethiazide $(0.5 \mathrm{mg})$. She had a past history of osteomyelitis at the age of nine, and she could not attend further elementary school education since that time. She was not good at reading or writing.

Her body temperature was $36.7^{\circ} \mathrm{C}$. Blood pressure was $160 / 95 \mathrm{mmHg}$, and pulse rate was $60 / \mathrm{m}$. Her skin was wet due to mild sweating. She was bedridden and mute. She could obey simple oral commands. On neurological examination, external eye movement was normal and nystagmus was not seen. Her face was oily and masked. The tongue

Department of Neurology, National Hospital Organization, Shiga Hospital, Japan

Received for publication September 7, 2010; Accepted for publication November 7, 2010

Correspondence to Dr. Kengo Maeda, maeda-kengo@shiga-hosp.jp 


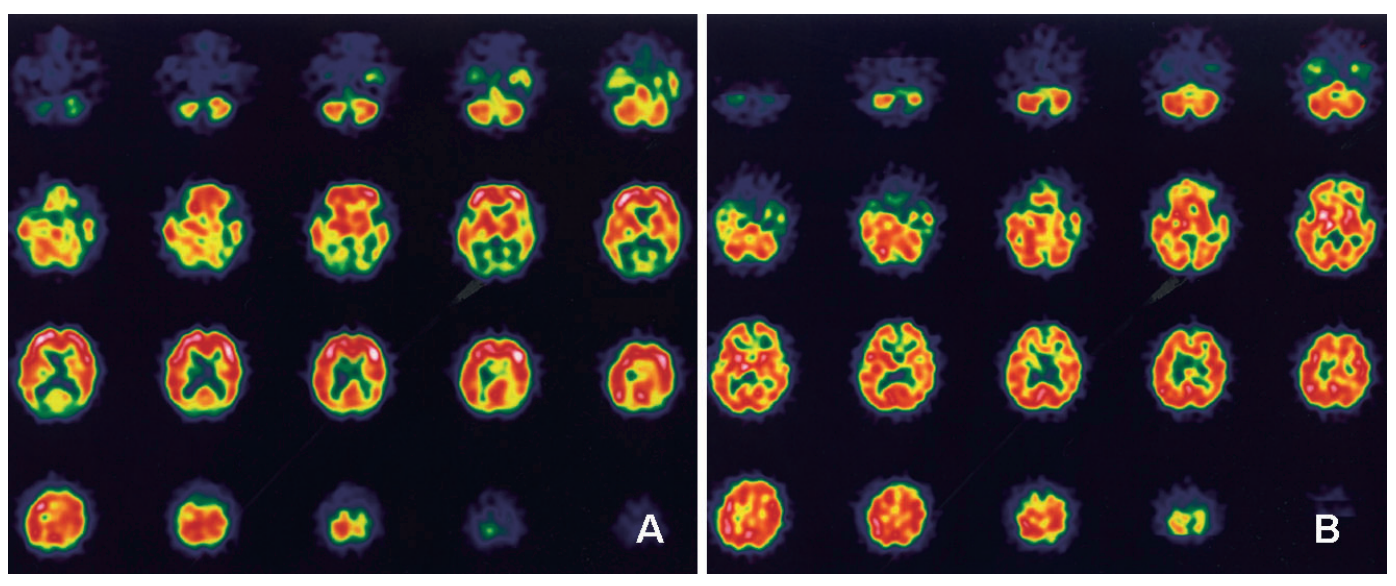

Figure 1. Single photon emission computed tomography using ${ }^{123} \mathrm{I}-\mathrm{N}$-isopropyl-P-iodoamphetamine. Cerebral blood flow was remarkably decreased in the bilateral occipital lobe before treatment (A). After treatment, occipital lobe hypoperfusion was improved (B).

was normal appearance. There was not dysphagia. All limbs were in flexion posture. She showed motiveless resistance to maintenance of rigid posture against attempts to be moved, suggesting catatonic rigidity. Resting tremor was observed in them. Deep tendon reflex was all normal. There was no pathological reflex. Detailed sensory examination, coordination, and cognitive examination was impossible.

Laboratory findings were normal except for a high level of LDL-cholesterol. Plasma creatine kinase was $44 \mathrm{IU} / \mathrm{L}$. Thyroid function was normal and GAD antibody was negative. Temporal and occipital lobes were not atrophic on magnetic resonance imaging (MRI). Electroencephalogram (EEG) showed low amplitude $8 \mathrm{c} / \mathrm{s}$ alpha waves in the occipital region. Myocardial scintigraphy using ${ }^{123} \mathrm{I}$ metaiodobenzyl guanidine (MIBG) showed a decreased uptake (early phase H/M 1.62, delayed phase H/M 1.34).

Quetiapine was decreased to $75 \mathrm{mg} /$ day on the admission day. Since psychosis was not deteriorated, quetiapine was discontinued. Donepezil was also stopped since there is a case report stating that donepezil could cause catatonia (6). SPECT using ${ }^{123} \mathrm{I}-\mathrm{N}$-isopropyl-P-iodoamphetamine revealed remarkable hypoperfusion in the bilateral occipital lobes and hyperperfusion in the bilateral frontal lobes (Fig. 1A). She did not eat foods because she was afraid that insects in her body develop. Nasogastric tube was inserted to start tube nutrition. Drip infusion test of 1-dopa was performed as previously reported (7). However, intravenous infusion of 1dopa had no effect on her motor dysfunction. Subsequently, oral administration of 1-dopa/carbidopa was started and gradually increased up to $500 \mathrm{mg} / \mathrm{day}$, which also resulted in failure. A case report which showed modifiedelectroconvulsive therapy (m-ECT) improved psychosis and occipital hypoperfusion in a single DLB patient (8) encouraged us to use antidepressant. Amitriptyline was administered at the initial dose of $10 \mathrm{mg} /$ day to $20 \mathrm{mg} /$ day. After two weeks from administration of amitriptyline, she began to speak spontaneously. Catatonic rigidity was also improved. The dose of amitriptyline was elevated to $60 \mathrm{mg} / \mathrm{day}$ and lorazepam was added orally from then at the dose of $1.5 \mathrm{mg} /$ day. Further two weeks later, she asked us to take a nasogastric tube off and declared to eat foods. The dose of lorazepam was decreased to $0.5 \mathrm{mg} /$ day due to daily sleepiness. She recovered to be able to eat foods with chopsticks and sometimes she talked with smiling. Cogwheel-like rigidity remained in her arms, but resting tremor was hardly seen. There was no nocturnal cry. Visual hallucination and delusion became minimal, and they did not disturb her daily life. Revised version of Hasegawa dementia scale which does not require reading or writing showed 14/30, which suggested that she has some type of dementia. The second SPECT showed marked improvement of perfusion of the frontal and occipital lobes (Fig. 1B). Cerebral blood flow of the frontal and occipital lobes was assessed to be normal when analyzed with easy Z-score Imaging System (9) (Fig. 2). The amplitude and frequency of alpha waves was increased in EEG. The clinical course is shown in Fig. 3.

\section{Discussion}

The patient was diagnosed as having probable DLB or Parkinson's disease with dementia (PDD) according to the diagnostic criteria (10). Results of SPECT and MIBG myocardial scintigraphy supported the diagnosis. Although the adverse effect of quetiapine could not be ruled out, her motor dysfunction was thought to be catatonia. Catatonia is diagnosed when there are at least two out of five major symptoms; motoric immobility, excessive motor activity, extreme negativism, peculiarities of voluntary movements, and echolalia/echopraxia (11). She showed motoric immobility and extreme negativism. Catatonia is thought to be caused by not only schizophrenia but also emotional disorders or general diseases (11). Catatonia in DLB patients have also been reported $(6,12,13)$. Catatonia in the present case was thought to be associated with depression which sometimes complicates DLB. A pharmacological approach to the psychiatric symptoms in DLB is sometimes difficult due to neu- 


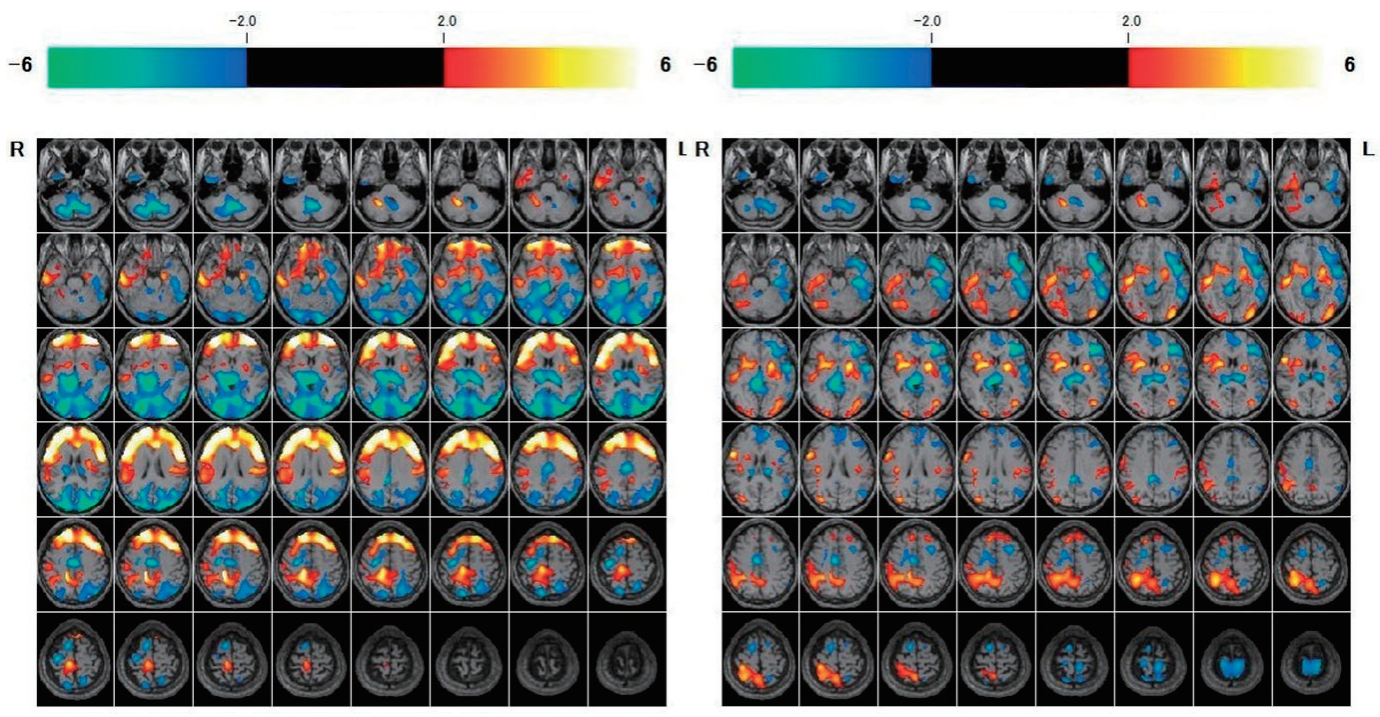

Figure 2. Data of cerebral blood flow (Fig. 1) analyzed with easy Z-score Imaging System. Left; before treatment, right; after treatment. Decreased cerebral blood flow under a Z-score of $>2$ points was indicated in the dark color, while increased cerebral blood flow over a $\mathrm{Z}$-score of $>\mathbf{2}$ points was indicated in the bright color.

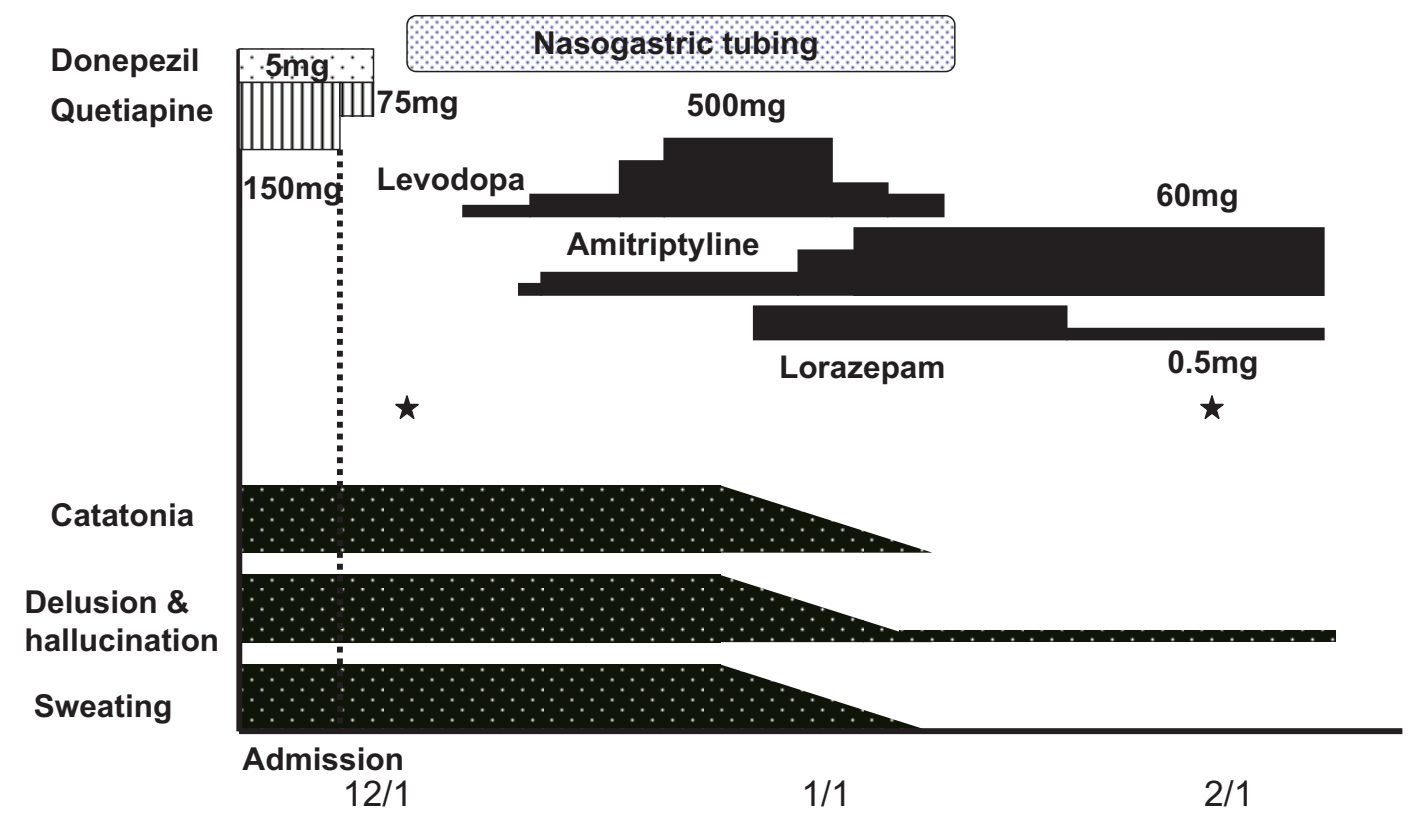

Figure 3. Clinical course. The asterisks mean SPECT. The first SPECT was performed six days after donepezil was stopped.

roleptic supersensitivity. Although the acetylcholinesterase inhibitor donepezil (3-5), quetiapine (14) and Yi-Gan San (15) were reported to be beneficial on psychiatric symptoms in DLB, all of them were not effective in this case. We first administered amitriptyline to this patient. Amitriptyline dramatically improved catatonia and psychiatric symptoms. Although we added low-dose lorazepam with amitriptyline, her symptoms began to improve before administration of lorazepam. There have been no large clinical trials on the effectiveness of antidepressants in DLB. Visual hallucination was reported to be improved with the selective serotonin reuptake inhibitor paroxetine in two patients (16). Neuro- chemical studies showed reductions in cortical serotonin (5HT) terminals in DLB, indicated by lower levels of both 5HT and 5-hydroxyindole-3-acetic acid $(17,18)$. Both amitriptyline and paroxetine enhance synaptic 5-HT effects. The present case suggests that an antidepressant might be effective for psychiatric symptoms in some DLB patients.

In addition to the clinical symptoms, occipital lobe hypoperfusion seemed to be normalized. The mechanism of occipital lobe hypometabolism and hypoperfusion in DLB remains to be clarified. Pathological study revealed that the density of Lewy bodies was the lowest in the occipital lobes (19). Taken together, our case and the patient treated 
with m-ECT (8) suggest that occipital lobe hypoperfusion might not be due to neurodegenerative changes but rather it is functional and reversible. Furthermore, occipital lobe hypoperfusion could be a marker of treatments in DLB. Although donepezil has been reported to increase occipital cerebral blood flow, the magnitude of the increase was limited to approximately $3 \mathrm{mls} / \mathrm{min}$ (20). As far as we know, there is no report which showed normalization of occipital cerebral blood flow in DLB with some treatment except for m-ECT therapy (8). Recently, frontal hyperperfusion was observed in DLB patients with delusion (21). In the present case, this abnormality was also improved.

In summary, although further study is needed to clarify whether the hypoperfusion of occipital lobes in DLB might be reversible or not, catatonia responsive to antidepressants or lorazepam should not be overlooked even in patients who appear to have an advanced stage of dementia (22).

The authors state that they have no Conflict of Interest (COI).

\section{References}

1. Kemp PM, Holmes C. Imaging in dementia with Lewy bodies: a review. Nucl Med Commun 28: 511-519, 2007.

2. Buracchio T, Arvanitakis Z, Gorbien M. Dementia with Lewy bodies: Current concepts. Dement Geriatr Cogn Disord 20: 306320, 2005.

3. Samuel W, Caligiuri M, Galasko D, et al. Better cognitive and psychopathologic response to donepezil in patients prospectively diagnosed as dementia with Lewy bodies: a preliminary study. Int J Geratr Psychiatry 15: 794-802, 2000.

4. Aarsland D, Bronnick K, Karlsen K. Donepezil for dementia with Lewy bodies: a case study. Int J Geriatr Psychiatry 14: 69-72, 1999.

5. Minett TS, Thomas A, Wilkinson LM, et al. What happens when donepezil is suddenly withdrawn? An open label trial in dementia with Lewy bodies and Parkinson's disease with dementia. Int J Geriatr Psychiatry 18: 988-993, 2003.

6. Morita S, Miwa H, Kondo T. A patient with probable dementia with Lewy bodies, who showed catatonia induced by donepezil: A case report. No To Shinkei 56: 881-884, 2004 (in Japanese, Abstract in English).

7. Black KJ, Carl JL, Hartlein JM, Warren SL, Hershey T, Perlmutter JS. Rapid intravenous loading of levodopa for human research: clinical results. J Neurosci Methods 127: 19-29, 2003.
8. Yano T, Ono Y, Mori H, Nagao S, Fujita Y, Kuroda S. A case of DLB with successful treatment of m-ECT accompanied by a remarkable improvement of occipital lobe hypoperfusions on SPECT. Rinsho Seishin Igaku 35: 1269-1274, 2006 (in Japanese, Abstract in English).

9. Kanetaka H, Matsuda H, Asada $\mathrm{T}$, et al. Effects of partial volume correction on discrimination between very early Alzheimer's dementia and controls using brain perfusion SPECT. Eur J Nucl Med Mol Imaging 31: 975-980, 2004.

10. McKeith IG, Dickson DW, Lowe J, et al. Diagnosis and management of dementia with Lewy bodies: third report of the DLB Consortium. Neurology 65: 1863-1872, 2005.

11. Taylor MA, Fink M. Catatonia in psychiatric classification: A home of its own. Am J Psychiatry 160: 1233-1241, 2003.

12. McKeith IG, Ballard CG, Harrison RW. Antipsychotic sensitivity to risperidone in Lewy body dementia. Lancet 346: 699, 1995.

13. Xiong GL, Palomino A, Kahn DR, Bourgeois JA. Antipsychotic induced catatonia: a case of probable dementia with Lewy bodies. J Neuropsychiatry Clin Neurosci 21: 472-473, 2009.

14. Fernandez HH, Trieschmann ME, Burke MA, Friedman JH. Quetiapine for psychosis in Parkinson's disease versus dementia with Lewy bodies. J Clin Psychiatry 63: 513-515, 2002.

15. Iwasaki K, Maruyama M, Tomita N, et al. Effects of the traditional Chinese herbal medicine Yi-Gan San for cholinesterase inhibitor-resistant visual hallucinations and neuropsychiatric symptoms in patients with dementia with Lewy bodies. J Clin Psychiatry 66: 1612-1613, 2005.

16. Tanaka M, Kita T. Paroxetine and improvement of visual hallucinations in patients with dementia with Lewy bodies. J Am Geriatr Soc 53: 732-733, 2005.

17. Ohara K, Kondo N, Ohara K. Changes of monoamines in postmortem brains from patients with diffuse Lewy body disease. Prog Neuropsychopharmacol Biol Psychiatry 22: 311-317, 1998.

18. Perry EK, Marshall E, Thompson $P$, et al. Monoaminergic activities in Lewy body dementia: relation to hallucinations and extrapyramidal features. J Neural Transm Park Dis Dement Sect 6: 167-177, 1993.

19. Gomez-Tortosa E, Irizarry MC, Gomez-Isla T, Hyman BT. Clinical and neuropathological correlates of dementia with Lewy bodies. Ann NY Acad Sci 920: 9-15, 2000.

20. Mori T, Ikeda M, Fukuhara R, Nestor PJ, Tanabe H. Correlation of visual hallucinations with occipital rCBF changes by donepezil in DLB. Neurology 66: 935-937, 2006.

21. Nagahama Y, Okina T, Suzuki N, Matsuda M. Neural correlates of psychotic symptoms in dementia with Lewy bodies. Brain 133: 557-567, 2010.

22. Alisky JM. Is the immobility of advanced dementia a form of lorazepam-responsive catatonia? Am J Alzheimers Dis Other Demen 19: 213-214, 2004.

(C) 2011 The Japanese Society of Internal Medicine http://www.naika.or.jp/imindex.html 